\title{
Spectral resolution of optical filters based on diffractive optical elements with non-uniform illumination
}

\author{
Hallvard Angelskår \\ hallvard.angelskar@fys.uio.no
}

Aasmund S. Sudbø

\author{
University of Oslo, Center for Materials Science and Nanotechnology, Department of Physics, PO Box \\ 1048 Blindern, N-0316 Oslo, Norway \\ University of Oslo, University Graduate Center at Kjeller, PO Box 70, N-2027 Kjeller, Norway
}

We study the transmission coefficient and the spectral resolution of optical filters based on a diffractive optical element (DOE), with a collimating lens, input and output fibers. We limit our study to filters that can be analyzed with a scalar (i.e., Fourier-optical) formalism, and specify requirements that the filter geometry has to satisfy for this formalism to give an accurate description. Finally, we study the trade-off between power loss and spectral resolution in DOE-based filters. [DOI: 10.2971/jeos.2008.08016]

Keywords: diffraction, Gaussian beam, micro-optics, Fourier optics, spectroscopy

\section{INTRODUCTION}

Diffractive optical elements (DOEs) have recently received increased attention as a low-cost component that can be used for spectral filtering within correlation spectroscopy [1]. A correctly patterned reflective surface can be used to focus and reflect light of a certain wavenumber in a certain direction. By rotating this DOE one can sequentially focus different wavenumbers onto a detector [2]. The introduction of [3] has a brief review of various applications of DOE-based filters.

Our main application is the design of DOE-based optical filters. An important requirement for this application is that the scalar approximation (i.e., Fourier optics [4]) can be used in the diffraction calculations, to make them practical in terms of computer run time. In [5], a general formalism for the analysis of DOE-based filters was developed. We extend this formalism, and show that practical DOE-based filters can be designed so that they can confidently be analyzed with the scalar approximation.

A system of movable micro-mirrors which allows control of both phase and amplitude was analyzed in [3] within the scalar field approximation. In [3] a matched DOE illumination was assumed, meaning that the field distribution at the source was assumed to produce an illumination of the DOE that for all wavenumbers was uniform over the DOE, and zero outside it. Such an aperture can be called an ideal aperture. Note that when discussing the choice of input aperture, we refer to the field distribution of the input of the system. In Section 2.4 of [3], the limitations of the ideal aperture approximation are discussed. Instead of assuming such an ideal aperture, the illumination is assumed to overfill the DOE. This can be achieved by having a small input field distribution. In this case a uniform illumination of the DOE is a good approximation, but the filter then has a large power loss, since most of the light from the source goes outside the DOE. Such a small aperture approximation can more easily be realized in practise than an ideal aperture. Below we also use the word aperture when referring to the actual fiber end faces of the input and output fibers, but the meaning should be clear in the context.

We report on our analysis of a DOE-based filter (see Figure 1) with more realistic input and output apertures than in [3, 5] and [6], but limited to a static DOE. Hence we extend the formalism of [5] and [6] and study how the transmission through the system is altered by allowing an illuminating field that varies over the DOE and even overfills or underfills the DOE. We have studied how a non-ideal illumination of the DOE and a finite-size output aperture affects the power transmission and the spectral resolution of the filter.

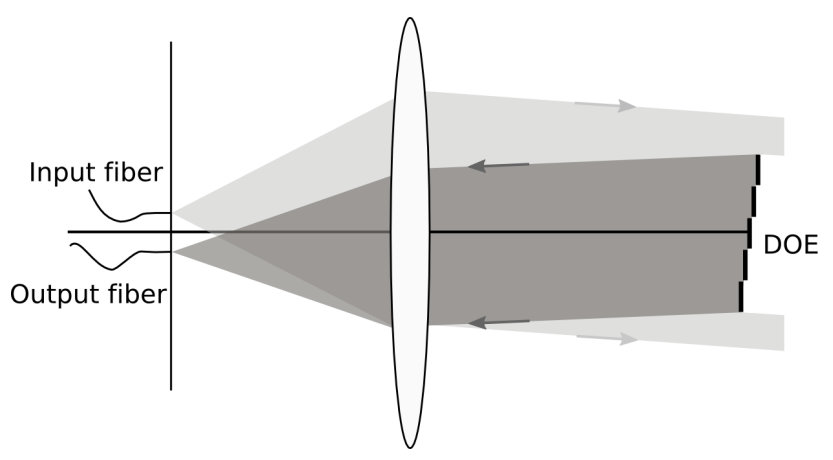

FIC. 1 The set-up with input and output fibers in the aperture plane, a collimating lens and an array of micro-mirrors functioning as the diffractive optical element.

In Section 2 we will briefly review the scalar theory of diffraction for a DOE filter, extending the analysis in [6] to the case of a realistic beam from an optical fiber. In Section 3 we apply the analysis to the monochromator, one of the most common DOE-based optical filters for laboratory use. For the monochromator example we establish a quantitative relation- 
ship between the power transmission and spectral resolution of the filter. Section 4 contains our main results, and Section 5 contains our conclusions.

\section{BACKGROUND: PROPAGATION THROUGH THE SYSTEM IN THE SCALAR APPROXIMATION}

We study the setup shown in Figure 2 with a collimating lens and input and output fibers, where the fiber end faces are labeled $\Gamma$ and $\Sigma$, respectively. The fiber end faces are called input and output apertures, and the plane they lie in is called the aperture plane. The setup resembles that of the Ebert-Fastie mount for monochromators, see for instance [7].

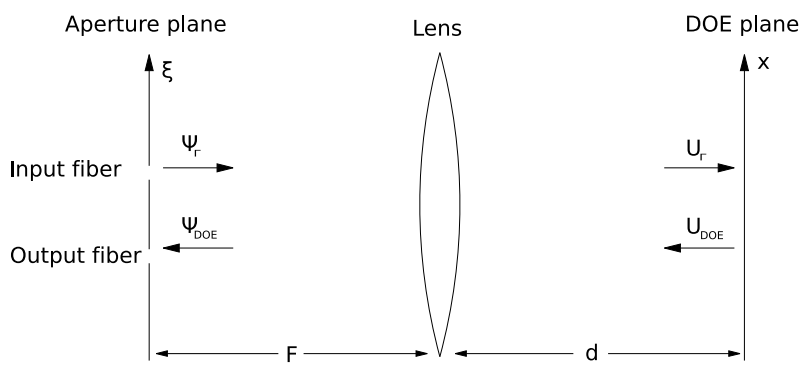

FIC. 2 The set-up with input and output fibers in the aperture plane, and a flat mirror in the DOE plane situated at a distance $d$ behind a collimating lens.

We first consider propagation of monochromatic light with the wavenumber

$$
v=1 / \lambda=f / c,
$$

where $\lambda$ is the vacuum wavelength, $c$ is the speed of light, and $f$ is the frequency. We use a standard formula from Fourier optics (see for instance Eq. (4.2-8) in [8] or Eq. (5-19) in [4]), following the sign conventions of [4] for the definitions of the forward and inverse Fourier transforms.

The field distribution $U_{D O E}(x, y, v)$ immediately after interacting with the DOE, see Figure 2, is related to the output field distribution $\Psi_{D O E}(\xi, \eta, v)$ by:

$$
\begin{aligned}
\Psi_{D O E} & (\xi, \eta, v)=\frac{v}{i F} \exp (-i 2 \pi v(F+d)) \\
& \times \exp \left(i \pi v(d-F) \frac{\xi^{2}+\eta^{2}}{F^{2}}\right) \\
& \times \iint_{D O E} U_{D O E}(x, y, v) \exp \left(i \frac{2 \pi v}{F}(\xi x+\eta y)\right) d x d y .
\end{aligned}
$$

For the propagation from the input aperture to the DOE plane, the distance in front of the lens is equal to the focal length $F$ and behind the lens it is equal to $d$. The propagation is then described by:

$$
\begin{aligned}
U_{\Gamma}(x, y, v) & =\frac{v}{i F} \exp (-i 2 \pi v(F+d)) \\
& \times \iint_{\Gamma} \Psi_{\Gamma}(\xi, \eta, v) \exp \left(i \pi v(d-F) \frac{\xi^{2}+\eta^{2}}{F^{2}}\right) \\
& \times \exp \left(i \frac{2 \pi v}{F}(\xi x+\eta y)\right) d \xi d \eta
\end{aligned}
$$

Here $\Psi_{\Gamma}(\xi, \eta, v)$ is the input field distribution, and $U_{\Gamma}(x, y, v)$ is the field distribution immediately in front of the DOE. The subscript DOE on the integral in Eq. (2) is meant to indicate that the integral is taken over the DOE plane, and the subscript $\Gamma$ in Eq. (3) that the integral is carried out over the aperture plane.

We assume that the system is designed with a geometry such that the exponential containing $(d-F)$ in Eq. (3) can be disregarded for

$$
0<d<2 F .
$$

For this assumption to be valid, we will have to place the input and output fibers as close to the optical axis as possible. The fiber mode field radius is small compared to the fiber radius, typically around $5 \mu \mathrm{m}$. Thus the maximum value taken by $\xi$ or $\eta$ in Eq. (3) where $\Psi_{\Gamma}(\xi, \eta, v)$ is non-negligible is approximately equal to the offset $R$ of the center of the source fiber relative to the optical axis. The offset $R$ must then fulfill the condition

$$
\pi v R \ll F / R
$$

If the fibers are placed as close as possible to one another and to the optical axis, the offset $R$ equals the fiber radius. A standard telecom fiber has a diameter of about $125 \mu \mathrm{m}$, so the offset can be made as small as $R=62.5 \mu \mathrm{m}$, and Eq. (5) then requires $F \gg 1 \mathrm{~cm}$ for a wavenumber of $1 \mu \mathrm{m}^{-1}$.

\subsection{Transfer function with a flat mirror in the DOE plane}

We start by considering a single flat mirror, and use the formalism in [6] but with zero tilt angle for the DOE. This is a simple, yet important case, because the transmission spectrum of a mirror represents an upper bound for the transmission spectrum of any DOE-based filter.

As in [6], we assume the input fiber is a single mode fiber in which a field of amplitude $e_{i n}(v)$ and mode field distribution $\phi_{\Gamma}(\xi, \eta)$ is propagating. $e_{i n}(v)$ is defined so that

$$
P=\int_{v}\left|e_{i n}(v)\right|^{2} d v
$$

is the total optical power propagating in the fiber, and $\left|e_{i n}(v)^{2}\right|$ is the power spectral density of the waves propagating in the fiber. Furthermore, we assume the input fiber to be centered on $\left(\xi=\xi_{\Gamma_{0}}, \eta=\eta_{\Sigma_{0}}\right)$. We can then define the coordinates

$$
\begin{gathered}
\xi_{\Gamma}=\xi-\xi_{\Gamma_{0}}, \\
\eta_{\Gamma}=\eta-\eta_{\Gamma_{0}} .
\end{gathered}
$$

The fiber mode field distribution $\phi_{\Gamma}$ is centered on $(0,0)$ in these coordinates. We assume the field coming out of the input fiber has the same distribution as the fiber mode:

$$
\Psi_{\Gamma}(\xi, \eta, v)=\Psi_{\Gamma}\left(\xi_{\Gamma}+\xi_{\Gamma_{0}}, \eta_{\Gamma}+\eta_{\Gamma_{0}}, v\right)=\phi_{\Gamma}\left(\xi_{\Gamma}, \eta_{\Gamma}, v\right) e_{i n}(v) .
$$

The input mode field $\phi_{\Gamma}$ is normalized according to

$$
\iint_{\Gamma} \phi_{\Gamma}^{2}\left(\xi_{\Gamma}, \eta_{\Gamma}, v\right) d \xi_{\Gamma} \eta_{\Gamma}=1
$$

The field incident on the DOE is given by Eq. (3). The interaction with the DOE is modeled by multiplying the field before 
the interaction with a complex reflection function $s(x, y, v)$, so that the field after the interaction is

$$
U_{D O E}(x, y, v)=U_{\Gamma}(x, y, v) s(x, y, v) .
$$

We can then propagate the $U_{D O E}(x, y, v)$ back to the aperture plane using Eq. (2). The field that couples into the output fiber can in this way be related to our input field $\Psi_{\Gamma}(\xi, \eta, v)$. In the same way as for the input fiber, we define the mode field distribution $\phi_{\Sigma}\left(\xi_{\Sigma}, \eta_{\Sigma}, v\right)$ of the output fiber with the coordinates

$$
\begin{aligned}
& \xi_{\Sigma}=\xi-\xi_{\Sigma_{0}}, \\
& \eta_{\Sigma}=\eta-\eta_{\Sigma_{0}} .
\end{aligned}
$$

The output fiber is also assumed to be single-mode over the whole spectral range of the input field $\Psi_{\Gamma}(\xi, \eta, v)$, and it is assumed to be normalized over the output aperture in the same way as the input fiber mode distribution (see the normalization in Eq. (9)). The amplitude of the field coupled into the output fiber is then given by

$e_{\text {out }}(v)=\iint_{\Sigma} \Psi_{D O E}\left(\xi_{\Sigma}+\xi_{\Sigma_{0}}, \eta_{\Sigma}+\eta_{\Sigma_{0}}, v\right) \phi_{\Sigma}\left(\xi_{\Sigma}, \eta_{\Sigma}, v\right) d \xi_{\Sigma} d \eta_{\Sigma}$

The transfer function is the ratio of the amplitudes of the field in the output fiber and the input fiber,

$$
\begin{aligned}
H(v) & =\frac{e_{\text {out }}(v)}{e_{\text {in }}(v)} \\
& =\iint_{D O E} s(x, y, v) u_{\Gamma}(x, y, v) u_{\Sigma}(x, y, v) d x d y,
\end{aligned}
$$

where we have defined the illumination fields $u_{\Gamma}(x, y, v)$ and $u_{\Sigma}(x, y, v)$ in the DOE plane:

$$
\begin{aligned}
u_{k}(x, y, v) & =\frac{v}{i F} \exp (-i 2 \pi v(F+d)) \iint_{k} \phi_{k}\left(\xi_{k}, \eta_{k}, v\right) \\
& \times \exp \left(i \frac{2 \pi v}{F}\left[x\left(\xi_{k}+\xi_{k_{0}}\right)+y\left(\eta_{k}+\eta_{k_{0}}\right)\right]\right) d \xi_{k} d \eta_{k}
\end{aligned}
$$

where $k$ is either $\Gamma$ or $\Sigma$. To arrive at Eq. (14) we left out the exponential factor containing $(d-F)$ in Eqs. (2) and (3) under the assumption that the inequalities in Eqs. (4) and (5) hold where $\phi_{\Sigma}\left(\xi_{\Sigma}, \eta_{\Sigma}, v\right)$ and $\phi_{\Gamma}\left(\xi_{\Gamma}, \eta_{\Gamma}, v\right)$ are non-negligible.

In the next section we introduce a Gaussian input fiber mode field and discuss the transfer function in this case, first for a flat mirror in the DOE plane, and then for a set of micromirrors acting as the DOE. We shall let the amplitude of the illuminating fields Eq. (14) vary over the DOE and also overfill or underfill the DOE.

\section{TRANSFER FUNCTION FOR A SYSTEM WITH GAUSSIAN FIBER MODE FIELD DISTRIBUTION}

We choose the mode field distribution for both the input fiber and the output fiber to be a Gaussian distribution

$$
\phi_{k}(\xi, \eta, v)=\frac{\sqrt{2}}{\sigma_{k}(v) \sqrt{\pi}} \exp \left(-\frac{\left(\xi^{2}+\eta^{2}\right)}{\left(\sigma_{k}(v)\right)^{2}}\right) .
$$

With $k=\Gamma$ this represents the mode field distribution of the input fiber, and with $k=\Sigma$ it represents that of the output fiber. A Gaussian mode field distribution allows us to perform analytic calculations, with a mode field distribution that is also a close approximation to the mode field distribution of standard step-index fiber at the operating wavenumber [9]. In such a fiber, the mode field diameter in general depends on wavenumber. See Section 4.2 for a discussion of the transfer function where a standard parametrization of this dependence is taken into account. By convention, see for instance [9], the term fiber mode radius when referring to a Gaussian field, is the distance from the center where the field strength has dropped by a factor $e$.

Let us assume that the detector is small and positioned so as to maximize the collection of light reflected by a DOE that is just a simple mirror. What a patterned DOE then can do compared to a mirror is to direct some of the optical power away from the detector. Hence, a DOE filter cannot transmit more power than a mirror, not for any frequency. The computed transmission spectrum for a simple mirror represents an upper limit for the transmission of a DOE with the same projected area as the mirror. This limiting mirror transmission spectrum therefore becomes an important characteristic of any practical DOE filter.

The transfer function for our setup is given by Eq. (13). We also define the power transmission $T(v)$ of the filter as the absolute square of the transfer function:

$$
T(v)=|H(v)|^{2} .
$$

For a flat mirror that is perfectly reflecting for all wavenumbers of interest, the complex reflection function is $s(x, y, v)=$ 1. Eq. (14) contain a two-dimensional inverse Fourier transformation of the fiber mode fields $\phi_{k}$, so the illumination fields with Gaussian mode field distribution become:

$u_{k}(x, y, v)=\frac{\sqrt{2 \pi} v \sigma_{k}(v)}{i F} \exp (-i 2 \pi v(F+d))$

$\times \exp \left(i \frac{2 \pi v}{F}\left[x \xi_{k_{0}}+y \eta_{k_{0}}\right]\right) \exp \left(-\frac{\pi^{2} v^{2}\left(\sigma_{k}(v)\right)^{2}}{F^{2}}\left[x^{2}+y^{2}\right]\right)$.

Here $k=\Gamma$ and $k=\Sigma$ labels the illumination field for the input and output fibers respectively.

\subsection{Transfer function for a flat, square mirror}

We consider a square mirror with side length $W$. The input and output fibers are assumed to be placed symmetrically about the optical axis, with center coordinates $\xi_{\Gamma_{0}}=-\xi_{\Sigma_{0}}$, and $\eta_{\Gamma_{0}}=\eta_{\Sigma_{0}}=0$. Furthermore, we assume in this section that the fiber mode field distributions are equal for the input and output fibers:

$$
\sigma_{\Gamma}(v)=\sigma_{\Sigma}(v)=\sigma(v)
$$

Inserting the expressions for the illuminating fields, Eq. (17) into Eq. (13), integrating over the square DOE, and using the following definition of the error function

$$
\operatorname{erf}(x)=\frac{2}{\sqrt{\pi}} \int_{0}^{x} e^{-t^{2}} d t
$$


yields

$$
H(v)=-\exp (-i 4 \pi v(F+d))\left(\operatorname{erf}\left[\frac{\pi v \sigma(v) W}{\sqrt{2} F}\right]\right)^{2} .
$$

The transfer function for the flat mirror will be used as a reference for the transmission coefficient of the monochromator setup we will study in the following (Section 3.3).

In Eq. (3) in [3], the expression for the transfer function (referred to there as the filtering function) is valid under the assumptions that the incident fields are uniform over the DOE, and that they are zero outside of the DOE. This is called the ideal aperture approximation, and is very hard to realize in practice. This approximation is discussed in section 2.4 in [3]. By assuming the less restrictive small aperture approximation, the fields can be taken to be uniform over the entire DOE, because the DOE is overfilled. This overfilling necessarily leads to loss of optical power, which can be accounted for by introducing correction factors as suggested in [3].

\subsection{Transfer function for a small DOE}

Assuming a small DOE size (or equivalently small wavenumber, small source or small detector) in Eq. (20) leads to a simple expression for the power transmission:

$$
T(v) \approx \frac{v^{4}}{F^{4}} A_{e f f, \Sigma} A_{e f f, \Gamma} A_{D O E}^{2} .
$$

The effective areas are defined by

$$
A_{e f f, k}=2 \pi \sigma_{k}^{2}
$$

for $k$ equal to $\Sigma$ for the output fiber and $\Gamma$ for the input fiber. The definition Eq. (22) of $A_{e f f, k}$ is chosen so that Eq. (21) is the optical power collected by a small incoherent detector with an area equal to $A_{e f f, \Sigma}$, centered in the output aperture.

Figure 3 shows the power transmission spectrum computed with the small-DOE approximation Eq. (21) compared to what the more accurate Eq. (20) yields. The small-DOE approximation agrees quite well with the general solution for power transmissions less than 0.1. As discussed above, the case of a flat mirror is important as an upper limit for the power transmission from a DOE of a given area. The simple small-DOE approximation, Eq. (21), is valuable in this context.

\subsection{Transfer function for a monochromator setup}

In this section we study an array of rectangular micro-mirrors (Figure 4), a model representing the planar diffraction grating often used in a monochromator. The mirrors that together make up the grating are assumed to be identical, and placed so that the projected area of the DOE on the $x y$-plane is symmetric about the origin of the xy-coordinate system. The DOE is divided in the $x$-direction into $N$ micro-mirrors which have the full width of the DOE in the $y$-direction. The height $h$ of each micro-mirror is the total width of the DOE divided by the total number of micro-mirrors:

$$
h=\frac{W}{N} .
$$

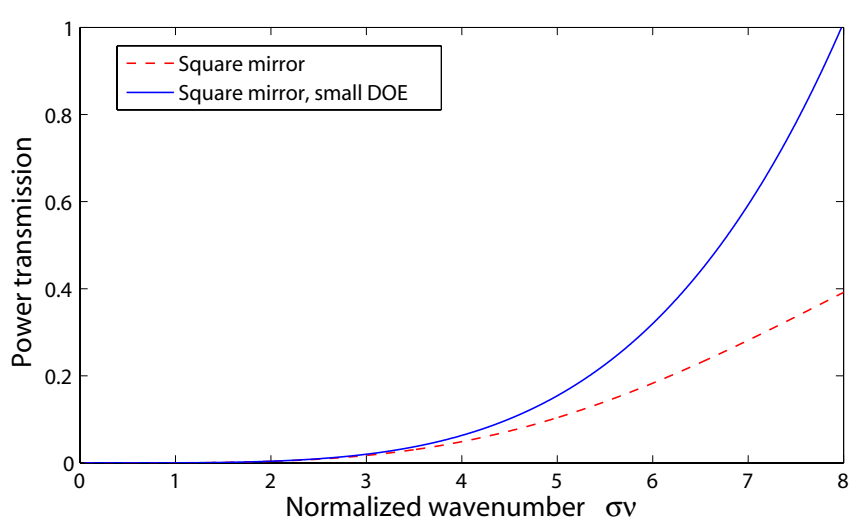

FIC. 3 Power transmission versus wavenumber for a flat, square mirror as the DOE from the general solution (red, dashed curve), Eq. (20), and in the approximation of small DOE (blue, solid), Eq. (21). We have assumed the fiber mode radius to be independent of wavenumber, and the input and output fibers to be identical, so that $\sigma_{\Gamma}=\sigma_{\Sigma}=5 \mu \mathrm{m}$. The normalized width of the mirror is $W / F=0.05$.

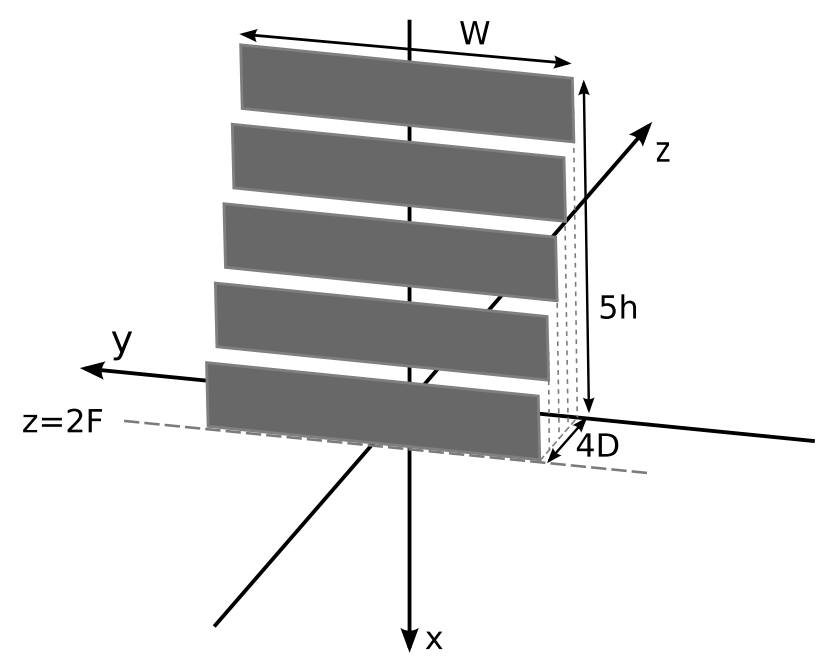

FIG. 4 The micro-mirror array that is used as a diffractive optical element in this setup. The $z$-distance between micro-mirrors is greatly exaggerated.

The mirrors are indexed from $n=1, \ldots, N$, and the bottom edge of each mirror is displaced a distance

$$
x_{n}=\left(-\frac{N}{2}+(n-1)\right) h,
$$

from the optical axis. The micro-mirrors are placed at distances $z_{n}$ behind the back focal plane of the lens. The front micro-mirror is placed at $z=2 F$ so that $z_{1}=0$, and the subsequent mirrors are placed at equal distances $D$ from one another. The distance $d_{n}$ from the lens to micro-mirror number $n$ is then:

$$
d_{n}=F+z_{n}, \quad \text { for } \quad z_{n}=(n-1) D .
$$

The monochromator system is chosen for simplicity and practical relevance, but qualitatively very similar results can be obtained for other DOE-based filter designs.

For the monochromator setup, we find the total transfer function by summing the transfer functions from all the micro- 
mirrors. We assume that the mirrors are independent and that they do not shadow one another. Thus we have

$$
H(v)=\sum_{n} H_{n}(v) \text {. }
$$

We use the transfer function for a single flat mirror (Eq. (20)) to find $H_{n}(v)$ for each micro-mirror. The extra distance $z_{n}$ for the light to travel from the focal plane to the micro-mirror only introduces a phase factor in the transfer function. Again, we assume that the filter geometry is such that the phase factor involving $(d-F)$ in Eqs. (2) and (3) can be disregarded, and then the phase factor is proportional to $d_{n}$. Hence, we obtain the transfer function $H_{n}(v)$ for a single micro-mirror at a distance $d_{n}$ from the lens:

$$
\begin{aligned}
H_{n}(v) & =-\frac{2 \pi v^{2}(\sigma(v))^{2}}{F^{2}} \exp (-i 8 \pi v F) \exp \left(-i 4 \pi v z_{n}\right) \\
& \times \iint_{n} \exp \left(-\frac{2 \pi^{2} v^{2}(\sigma(v))^{2}\left(x^{2}+y^{2}\right)}{F^{2}}\right) d x d y .
\end{aligned}
$$

Subscript $n$ on the integral indicates that the double integral is taken over micro-mirror number $n$. The power transmission for the monochromator system is then:

$$
\begin{aligned}
T(v)= & \left|\sum_{n} H_{n}(v)\right|^{2}=\frac{1}{4} \operatorname{erf}\left(\frac{\pi v \sigma(v) W}{\sqrt{2} F}\right)^{2} \mid \sum_{n} \exp \left(-i 4 \pi v z_{n}\right) \\
& \times\left.\left[\operatorname{erf}\left(\frac{2 \pi v \sigma(v) x_{n+1}}{\sqrt{2} F}\right)-\operatorname{erf}\left(\frac{2 \pi v \sigma(v) x_{n}}{\sqrt{2} F}\right)\right]\right|^{2}
\end{aligned}
$$

\section{RESULTS AND DISCUSSION}

We study the transmission and spectral resolution for a filter with a DOE consisting of an array of identical micro-mirrors illuminated by a Gaussian beam. The analysis is based on Fourier optics, which puts some constraints on the geometry of the filter system: The size of the DOE must be much less than the focal length of the lens: $W \ll F$. The source and detector apertures must be close to the optical axis, and their dimensions must be many times larger than the operating wavelength of the filter. Furthermore, the dimensions of the micro-mirrors must also be much larger than the operating wavelength. In effect, the wavenumber cannot be too small.

The expression for the power transmission of the monochromator system is given in Eq. (28). Let us for definiteness assume that the fiber mode radius $\sigma$ is $5 \mu \mathrm{m}$, and independent of wavenumber:

$$
\sigma(v)=\sigma,
$$

for both input and output fiber. We have made an exception in Section 4.2, where we introduce a standard parametrization of the wavenumber dependence of the fiber mode radius for a single-mode telecom fiber.

All the following results are for DOEs with a spacing in the $\mathrm{z}$-direction (along the optical axis) between micro-mirrors of

$$
D=M \frac{\lambda_{c}}{2}
$$

where $M$ is an integer, and in the figures the center wavelength was chosen to be $\lambda_{c}=1.5 \mu \mathrm{m}$. For a DOE with $N$ micro-mirrors, the total depth of the array is then $(N-1) D$ (see Figure 4).

As one can see from the expression for the illumination fields, Eq. (17), these have a Gaussian distribution in the DOE plane. The distribution is dependent on the wavenumber. In Figure 5 we have plotted the irradiance of the illumination fields in the DOE plane for four different wavenumbers. This shows how the field varies over the DOE for different wavenumbers. For small wavenumbers the DOE is almost uniformly illuminated, but a large portion of the beam misses the DOE, resulting in power loss. For large wavenumbers the beam effectively illuminate only the central part of the DOE. This leads to reduced spectral resolution of the filter.

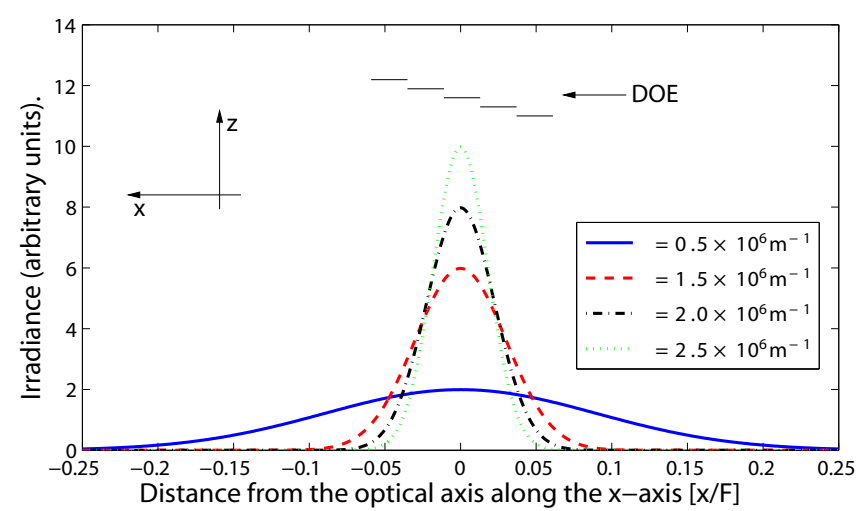

FIG. 5 The irradiance at the DOE plotted against distance from the optical axis in the $x$-direction, for various values of the wavenumber $v$. Above the graph is shown schematically an array of total width $W / F=0.1$, consisting of 5 identical micromirrors.

The illumination of the output aperture is shown schematically for light of two different wavenumbers in Figure 6. The angular divergence $\Delta \theta_{\text {div }}$ of the beam reflected from the DOE is inversely proportional to the diameter of the beam when it leaves the DOE. This diameter is equal to the DOE width $W$ for a small DOE, whereas for a large DOE, an effective beam diameter has to be computed from the power distribution of the illuminating beam. When the filter operates at the design wavenumber, the center of the beam hits the center of the output aperture. A monochromator has the property that when the wavenumber changes with $\Delta v$, the center of the beam shifts an angle $\Delta \theta_{\text {disp }}$. The resolution of the filter depends on how fast the beam is shifted away from the detector when the wavenumber is changed. The angular shift is inversely proportional to the range $\Delta z$ of positions $z_{n}$ covered by the illuminating beam. $\Delta z$ is equal to the total range of $z_{n}$ for a small DOE, whereas for a large DOE, an effective range has to be computed from the power distribution of the illuminating beam. We shall in the following see how the resolution and power transmission is affected by the geometry of the system.

\subsection{Varying the DOE geometry}

In Figure 7 we plot the power transmission for two DOEs of different size, each consisting of five identical micro-mirrors spaced at distances $D=\lambda_{c} / 2$ from one another. In the 


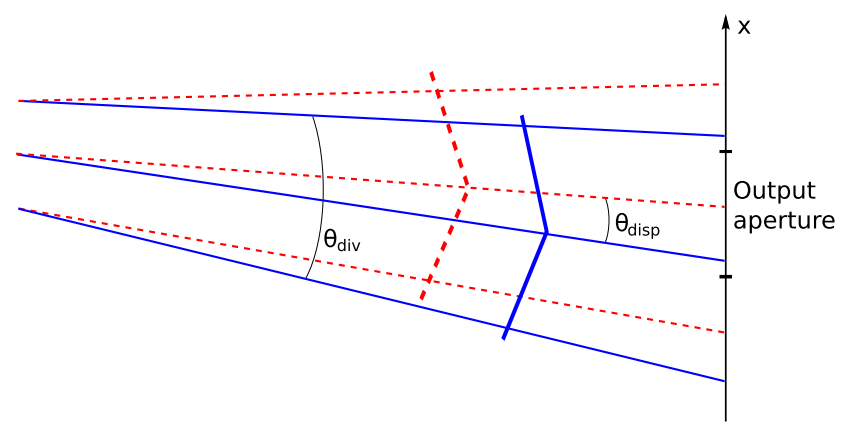

FIC. 6 Divergent beams resulting from the reflected field at the DOE shown for two different wavenumbers. The figure is intended to illustrate the far-field angular distribution of the reflected beam from the DOE, in the absence of the focusing lens in Figure 6. In general, the far-field distribution depends on the wavenumber, and the figure shows the special case of a change in wavenumber resulting in an angular shift $\Delta \theta_{\text {disp }}$ of the center of the beam. The beam has an angular divergence $\Delta \theta_{\text {div }}$ and the center of the beam shifts an angle $\Delta \theta_{\text {disp }}$ when the wavenumber changes by $\Delta v$.

same plots we show the same quantity for a flat mirror (red, dashed curve) as a reference showing the maximum power transmission for a DOE of the same size, as discussed above in Section 3. We have peaks in the power transmission for wavenumbers that are integer multiples of the design wavenumber $\left(\sigma v_{c} \approx 3.33\right)$. With increasing wavenumber, the peaks broaden and the peak transmission increases to one. This is more pronounced in Figure $7 \mathrm{~b}$ than in Figure $7 \mathrm{a}$, since Figure $7 \mathrm{~b}$ shows results for larger mirrors than Figure 7a.

The spectral resolution of the filter can be improved by increasing the range $\Delta z$ of mirror positions $z_{n}$, for example by increasing the number of mirrors and reducing the mirror size. However, for our main application, filter design, this is not straightforward, since not only the filter resolution, but the overall response function of the filter is affected by such a subdivision. This is not necessarily desired as it distorts the spectral filter.

In Figure 8 we plot the half-width versus power transmission for several systems with different number of mirrors but with equal total DOE width $W$. We have normalized the value of the half-widths to the number of micro-mirrors divided by the distance between two peaks in the diffraction pattern. Note however that as one approaches a large number of mirrors within a certain total DOE width $W$, one is approaching the domain where the micro-mirror width is no longer large compared to the wavenumber, and hence the scalar diffraction theory is no longer valid.

The normalized curves for different number of mirrors are very similar, showing that the trade-off between resolution and filter transmission is nearly the same in all cases. For instance we see that if we only allow a reduction of about $25 \%$ of the signal power, we must accept an increase in half-width of about $25 \%$.

This example shows that one must take care when designing such a filter with non-uniform illumination, and points out the important effects. We can make the same kind of statements
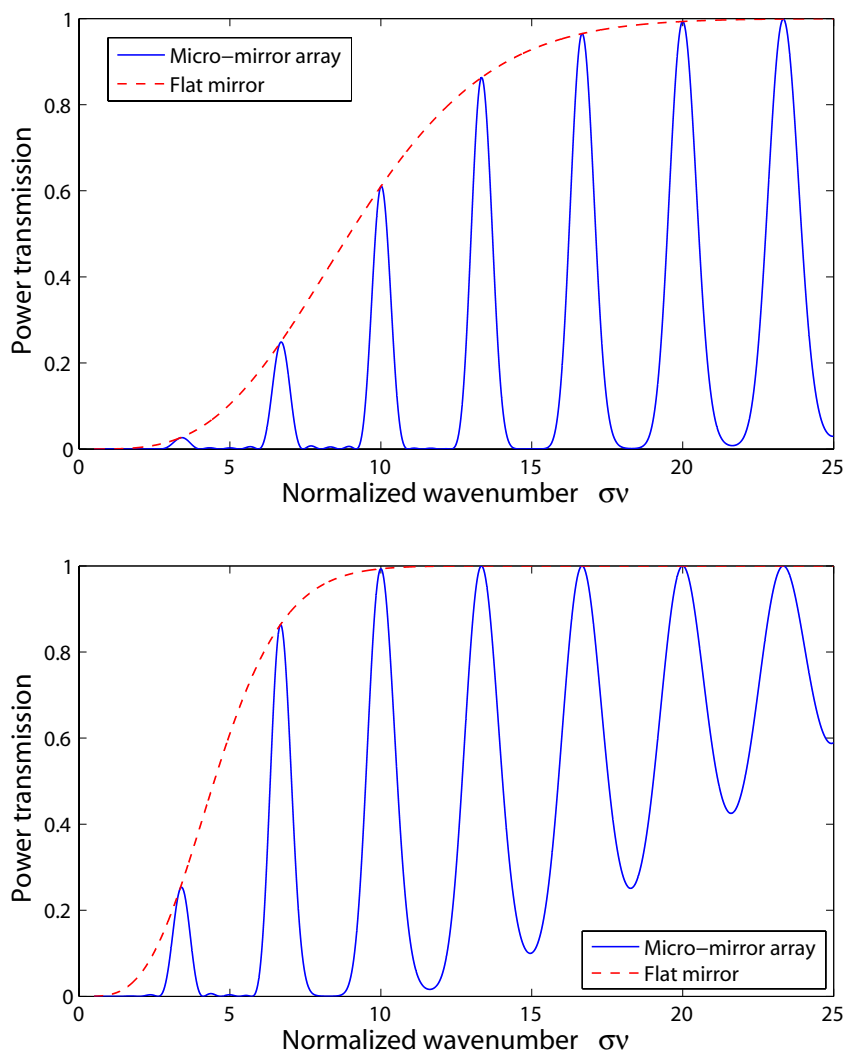

FIG. 7 Power transmission for a flat mirror (red, dashed) and a micro-mirror array (blue, solid) consisting of 5 micro-mirrors spaced at distances $D=\lambda_{c} / 2$ from one another. In (a) the width and height of the DOE is $W / F=0.05$ where $F$ is the focal length of the lens. In (b) we have the same plot but for a DOE with sides of length $W / F=0.1$.

regarding the trade-off between power transmission and spectral resolution for any DOE-based filter, as long as the filter geometry allows us to use Fourier optics.

\subsection{Wavenumber dependence of the mode field size}

As described in [9], the size of the fiber mode field distribution can be parametrized by

$$
\sigma(v)=a\left(0.65+1.619 V(v)^{-3 / 2}+2.879 V(v)^{-6}\right)
$$

where $a$ is the fiber core radius, and the $\mathrm{V}$ parameter is

$$
V(v)=2 \pi v a \sqrt{n_{1}^{2}-n_{2}^{2}} .
$$

Here $n_{1}$ is the refractive index in the fiber core, while $n_{2}$ is the index of the cladding. The parametrization is valid for $1.2<V<2.4$. In typical single-mode fibers for optical communication, $a=4 \mu \mathrm{m}$ and $V \approx 2.4$ for $v=1.2 \mu \mathrm{m}$, so that the parametrization is valid for $0.4 \mu \mathrm{m}^{-1}<v<0.8 \mu \mathrm{m}^{-1}$. The effective width of the power illuminating the DOE when Eq. (31) is used for $\sigma(v)$, has a maximum for $v=0.65 \mu \mathrm{m}^{-1}$. The power transmission in this case is shown in Figure 9 for a mirror of size $W / F=0.1$. We see that there is a minimum around $0.65 \mathrm{~mm}^{-1}$, corresponding to the maximum width of the illumination. 


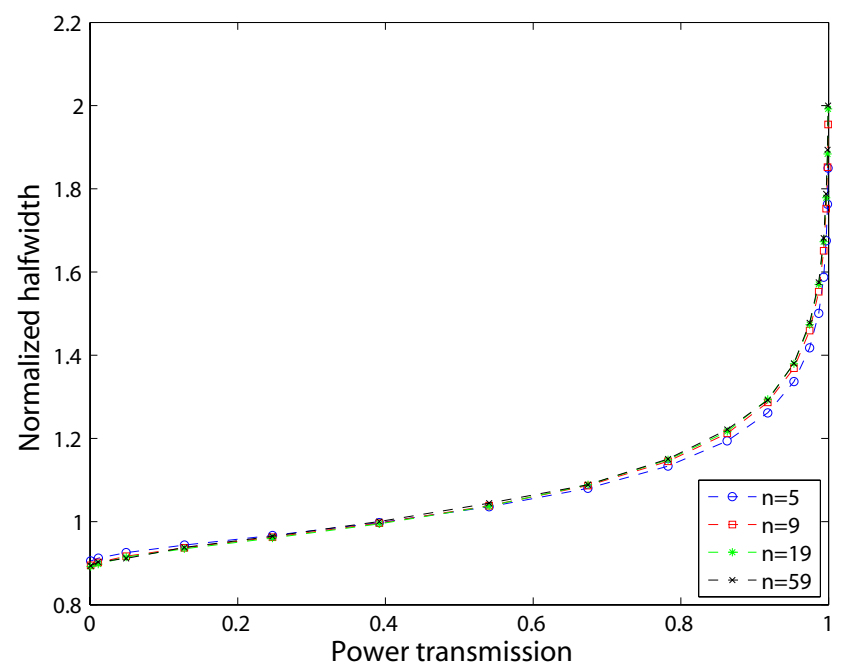

FIG. 8 Normalized half-width of the peaks in the transmission spectrum versus peak power transmission for DOEs with different numbers of micro-mirrors. The half-width is normalized by multiplying with the number of micro-mirrors and dividing by the distance between two peaks. The plot is for a DOE with sides $W / F=0.1$. The distance between micro-mirrors is $D=5 \lambda_{c} / 2$, chosen to achieve a suitable number of peaks in the power transmission(and thus data points in the plot).

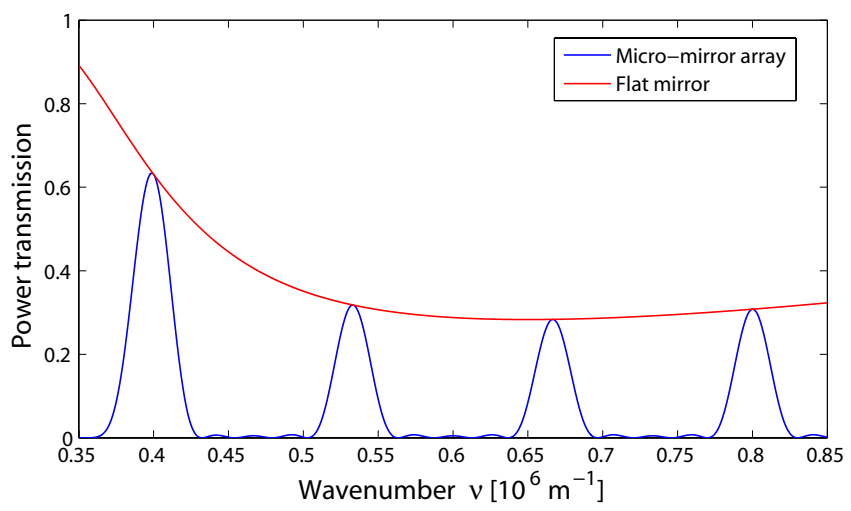

FIG. 9 Power transmission when the fiber mode field parametrization is taken into account. Plotted for a DOE with sides of $W / F=0.1$. We have also chosen to plot this for a distance between micro-mirrors of $D=5 \lambda_{c} / 2$ to produce a transmission spectrum with peaks on the interval where the parametrization is valid.

\section{CONCLUSION}

In many DOE applications a uniform illumination of the DOE is desirable. To achieve this in practice, one has to use an illuminating beam with an effective diameter that is larger than the DOE, overfilling the DOE, so that part of the incident power is lost. On the other hand, underfilling of the DOE to achieve high power transmission leads to poor spectral resolution for DOE-based filters.

By use of scalar diffraction theory we have found analytical expressions for the transfer function of DOE-based optical filters in general, considering the effect that a source and a detector with finite size have on the spectral response. We are reminded of the properties of a filter where a simple flat mir- ror is used as a DOE, in particular that its spectral response is not flat, and that the mirror spectrum represents an upper limit for the transmission spectrum of any DOE-based filter. We consider in particular a model of a monochromator. In part of our analysis, we take into account the wavenumber dependence of the waveguide modes of the input and output fibers.

Our main result is an estimate of spectral distortion introduced by a requirement for a small transmission loss in the micro-mirror array. Small transmission loss means a nonuniform illumination of the DOE, and hence leads to a distortion of the spectrum. For the specific case of a monochromator with a Gaussian beam illumination of the diffraction grating, if less than $25 \%$ transmission loss is required, more than $25 \%$ loss in spectral resolution must be accepted, regardless of the number of grooves in the grating.

\section{Acknowledgements}

This work was funded by the Norwegian Research Council under the project 174429/i40 "Micro-optical sensors for spectrometric applications". We would like to acknowledge Olav Solgaard for pointing out the need for a model of DOE-based optical filters that takes the receiver aperture into account. We would also like to thank Ruslan Belikov, Matthieu Lacolle, and Olav Solgaard for many helpful discussions on diffractive optical filters.

\section{References}

[1] M. B. Sinclair, M. A. Butler, A. J. Ricco, and S. D. Senturia, "Synthetic spectra: a tool for correlation spectroscopy" Appl. Optics 36, 3342-3348 (1997).

[2] 0. Løvhaugen, I.-R. Johansen, K. A. H. Bakke, B. G. Fismen, and S. Nicolas, "Dedicated spectrometers based on diffractive optics: design, modelling and evaluation" J. Mod. Optic. 51, 2203-2222 (2004).

[3] M. Lacolle, R. Belikov, H. Sagberg, 0. Solgaard, and A. S. Sudb $\phi$, "Algorithms for the synthesis of complex-value spectral filters with an array of micromechanical mirrors" 0pt. Express 14, 12590-12612 (2006).

[4] J. W. Goodman, Introduction to Fourier optics (2nd ed. McGrawHill, 1996).

[5] R. Belikov, Diffraction-Based Optical Filtering: Theory and Implementation with MEMS (Doctoral dissertation, Stanford University, UMI Dissertation Publishing, 2005).

[6] M. Lacolle, Optical spectral filtering with segmented diffractive optical elements (Doctoral dissertation, University of Oslo, Unipub, 2006).

[7] C. Palmer, Diffraction grating handbook (6th ed., Newport Corporation, 2005).

[8] B. E. A. Saleh and M. C. Teich, Fundamentals of photonics (John Wiley a Sons, Inc., 1991).

[9] G. P. Agrawal, Fiber-Optic Communication Systems (2nd ed., by John Wiley \& Sons, Inc., 1997). 